\title{
Desempenho de bovinos machos de origem leiteira submetidos a diferentes estratégias alimentares na recria e terminação
}

\section{Performance of dairy steers subjected to different food strategies during the growing and finishing phases}

\author{
Marcondes Dias de Freitas Neto ${ }^{1 *}$; Juliano José de Resende Fernandes ${ }^{2}$; \\ João Restle ${ }^{3}$; João Teodoro Pádua ${ }^{2}$; Pedro Leonardo de Paula Rezende \\ Fabrícia Rocha Chaves Miotto ${ }^{5}$ Kíria Karolline Gomes Moreira ${ }^{6}$
}

\begin{abstract}
Resumo
Objetivou se avaliar o desempenho de bovinos machos mestiços de origem leiteira, em confinamento, previamente mantidos com níveis de suplementação energética em pastagem de Brachiaria brizantha. Os tratamentos avaliados foram: TAA - "alto nível de ingestão de energia nas fases de recria e terminação"; TAM - "alto nível de ingestão de energia na fase de recria e médio na fase de terminação"; TMA - "médio nível de ingestão de energia na fase de recria e alto na fase de terminação" e TMM "médio nível de ingestão de energia nas fases de recria e terminação". Durante a fase de crescimento os animais receberam suplementação energética de $1,0 \%$ e $0,5 \%$ do peso corporal. Na fase de terminação os animais receberam dietas contendo $50 \%$ (nível médio de energia, NDT $=71 \%$ ) ou $80 \%$ (nível alto de energia, NDT $=80 \%$ ) de concentrado na matéria seca da dieta total. Os animais dos tratamentos TAA e TAM apresentaram peso corporal médio superiores no início do confinamento, devido a isso alcançaram pesos médios de abate com 42 e 21 dias a menos, respectivamente, quando comparados aos demais tratamentos. Nos tratamentos TAA e TAM, os animais também apresentaram maior consumo $(\mathrm{P}<0,05)$ de matéria seca $\left(\% \mathrm{PV}\right.$ e $\left.\mathrm{g} / \mathrm{PV}^{0,75}\right)$, ganho em peso médio diário e a conversão alimentar. Os animais suplementados com maior nível de energia na fase de recria apresentaram maior $(\mathrm{P}<0,05)$ perímetro torácico no inicio e menor ganho durante o confinamento. Não houve diferença $(\mathrm{P}>0,05)$ entre os tratamentos no final da terminação. $O$ alto nível de suplementação energética durante a fase de recria possibilitou aos animais um maior peso de entrada no confinamento, e consequentemente, melhor desempenho destes.
\end{abstract}

Palavras-chave: Confinamento, nível de concentrado, suplementação

\footnotetext{
Abstract

The objective of this study was to evaluate the performance of dairy crossbreed steers, in feedlot, previously fed with different supplemented energetic systems and Brachiaria brizantha pasture. The treatments were: TAA - high level intake of energy during the growing and finishing phases; TAM high intake of energy during the growing phase and medium intake of energy during of the finishing phase; TMA - medium intake of energy during growing phase and high intake of energy during finishing

${ }^{1}$ Bolsista de Pós-Doutorado, Universidade Federal da Bahia, UFBA, Salvador, BA. E-mail: marcondes_dias@hotmail.com

2 Profs. da Escola de Veterinária e Zootecnia da Universidade Federal de Goiás, EVZ/UFG, Goiânia, GO. E-mail: julianojrf@ gmail.com; teodoro@prppg.ufg.br

3 Prof. convidado, Universidade Federal do Tocantins, UFT, Araguaína, Tocantins, TO. E-mail: jorestle@terra.com.br

${ }^{4}$ Dr., Agência Goiana de Defesa Agropecuária, AGRODEFESA, Jataí, GO. E-mail: pedrozootec@hotmail.com

5 Prof ${ }^{a}$, UFT, Araguaína, TO. E-mail: fabriciarchaves@hotmail.com

${ }^{6}$ Discente de Doutorado, UFG, Goiânia, GO. E-mail: kiriakarolline@hotmail.com

* Autor para correspondência
} 
phase; TMM - medium intake of energy during the growing and finishing phases. During the growth phase the animals received supplementation of $1.0 \%$ and $0.5 \%$ body weight. In the finishing phase, the animals were fed diets containing $50 \%$ of concentrate (medium intake of energy, TDN $=71 \%$ ) or $80 \%$ of concentrate (high intake of energy, TDN $=80 \%$ ) in the dry matter of the total diet. The animals of the TAA and TAM treatments had higher average body weight at the beginning of feedlot due to that achieved average slaughter weights of 42 and 21 days less than the other treatments. The animals of the TAA and TAM treatments had higher dry matter intake $(\mathrm{P}<0.05), \% \mathrm{PV}$ and $\left.\mathrm{g} / \mathrm{PV}^{0}, 75\right)$, average daily gain and feed conversion than the other treatments. The animals supplemented with higher energy level in the growing phase had higher heart girth $(\mathrm{P}<0.05)$ and lower gain of this measure during the feedlot. There was no difference $(\mathrm{P}>0.05)$ between treatments for these measures at the end of the feedlot. The high level of energy supplementation during the growing phase allowed animals to a greater initial weight of the feedlot, and consequently, the best performance.

Key words: Feedlot, concentrate level, supplementation

\section{Introdução}

A bovinocultura leiteira no Brasil tem crescido e alcançado altos índices de desenvolvimento graças ao avanço tecnológico na área, exemplificado pelo aumento da utilização de ordenhadeiras mecânicas e inseminação artificial, entre outras. No entanto, apesar do expressivo desenvolvimento, o preço pago pelo litro de leite aos produtores brasileiros é pouco atrativo comprometendo a lucratividade da atividade leiteira. Dessa forma, para que o produtor consiga permanecer na atividade de maneira eficiente, é necessário buscar fontes de renda alternativas como a utilização dos bezerros machos para produção de carne.

Em geral, para maioria dos produtores de leite, os machos são indesejáveis, pois necessitam ingerir quantidades significativas de leite na fase inicial do seu desenvolvimento. Além disso, a forma como são criados, geralmente faz com que atinjam condições de abate com idade avançada, permanecendo mais tempo na propriedade, concorrendo com novilhas e vacas lactantes por pasto, mão de obra e alimentos concentrados.

No entanto, o não aproveitamento do bezerro macho para produção de carne faz com que o produtor de leite deixe de lado uma importante fonte de renda para seu sistema de produção, já que em países com pecuária de leite avançada, como Estados Unidos, Austrália e Nova Zelândia, a contribuição de bovinos de origem leiteira para produção de carne é elevada. Dessa forma, o Brasil deixa de produzir milhares de toneladas de carne por ano pelo não aproveitamento do bezerro leiteiro como animal destinado ao abate ou pelo manejo alimentar inadequado quando esta categoria é utilizada.

Para que a produção desses mestiços de origem leiteira se torne uma atividade rentável é necessário considerar todas as suas fases de criação, indo desde o nascimento até o abate. Para isso, é importante a escolha da melhor estratégia alimentar em cada etapa de criação, sendo que do nascimento à desmama o manejo será o mesmo que o das fêmeas, já durante a fase de recria o uso de suplementação múltipla permite aumentar o consumo de nutrientes digestíveis, melhorando assim a produtividade e a eficiência alimentar, resultando em maior peso na entrada do confinamento e permitindo que os animais atinjam mais rapidamente o peso e o acabamento de carcaça exigida pelos frigorificos (PORTO et al., 2008).

Objetivou-se com o presente trabalho verificar qual melhor estratégia de alimentação sobre o desempenho de bovinos machos mestiços de origem leiteira na recria e terminação.

\section{Material e Métodos}

O experimento foi conduzido do dia 23 de fevereiro a 29 de novembro de 2008 na cidade de Goiânia, com altitude média de $771 \mathrm{~m}$, latitude $16^{\circ}$ 36' Sul e longitude $49^{\circ} 15^{\prime}$ 'Oeste. A precipitação 
pluviométrica média durante todo o período experimental foi de $1072 \mathrm{~mm}$, com temperatura média de $23,43{ }^{\circ} \mathrm{C}$ e umidade relativa do ar média de $70,79 \%$. Os dados meteorológicos foram obtidos na estação meteorológica da Escola de Agronomia (EA/UFG, 2009).

Os dados foram analisados em delineamento inteiramente casualizado, em esquema fatorial 2x2 (dois níveis de suplementação na recria e duas proporções de concentrado na terminação), pelo seguinte modelo matemático:

$$
\mathrm{Y}_{\mathrm{ijk}}=\mu+\mathrm{a}_{\mathrm{i}(1,2)}+(\mathrm{ab})_{\mathrm{ij}}+\mathrm{b}_{\mathrm{j}(1,2)}+\mathrm{E}_{\mathrm{ijk}}
$$

Em que:

$\mathrm{Y}_{\mathrm{ijk}}=$ conjunto de observações da variável dependente correspondente a recria $i$, terminação $j$ e repetição $\mathrm{k} ; \mu=$ média de todas as observações; $\mathrm{a}_{\mathrm{i}}=$ efeito da i-esimo nível de suplementação na recria, sendo $1=0,5$ e $2=1,0 \%$ do peso vivo; $b_{j}=$ efeito da j-esima proporção de concentrado na MS da dieta na terminação, sendo $1=50$ e $2=80 \%$; (ab) ij = efeito da interação entre nível de suplementação na recria de ordem i e proporção de concentrado na terminação de ordem $\mathrm{j} ; \mathrm{E}_{\mathrm{ijk}}=$ erro experimental referente à observação do efeito da suplementação na recria i, proporção de concentrado na terminação j e repetição $k$.

Foram utilizados 24 bovinos machos mestiços de origem leiteira, adquiridos de fazendas produtoras de leite no estado de Goiás, com dez meses de idade, peso médio de $118 \pm 18 \mathrm{~kg}$ e 2,4 pontos de escore de condição corporal (ECC), conforme Restle (1977), no início do experimento, quando os animais estavam no pasto. Nessa fase os animais foram desverminados e vacinados segundo o calendário profilático da região.

$\mathrm{Na}$ fase de recria os animais foram mantidos em pastagem de Brachiaria brizantha e divididos em dois sistemas de suplementação: alto e médio, determinados em função do fornecimento equivalente a 1,0 e $0,5 \%$ do peso vivo (PV) de suplemento energético, na base da matéria seca
(MS), respectivamente. Os ingredientes utilizados na composição do suplemento durante a recria foram: grão de milho moído e mistura mineral, balanceados para atender as exigências do NRC (1996).

Após a recria os animais foram confinados com idade média de 15 meses e peso médio de 242,98 $\pm 38,07 \mathrm{~kg}$. As instalações eram compostas por baias individuais de 4 metros de comprimento por 2 metros de largura, totalizando $8 \mathrm{~m}^{2}$ por animal, parcialmente cobertas, providas de bebedouros em número de um para duas baias e cochos de alimentação individuais de mesma largura da baia, permanecendo até que os animais atingissem aproximadamente $390 \mathrm{~kg}$.

No confinamento todos os animais começaram o experimento recebendo uma dieta com $50 \%$ de concentrado e após sete dias, dois grupos de animais, sendo um que estava em alta suplementação a pasto e outro com média suplementação, passaram a receber uma dieta de $65 \%$ de concentrado. Após mais sete dias receberam a dieta final com $80 \%$ de concentrado, terminando assim, o período de adaptação de 14 dias. Sendo assim, cada lote da recria foi subdividido em dois sistemas de alimentação, com seis animais cada, recebendo alta $(80 \%)$ e média (50\%) proporção de concentrado na MS da dieta total, resultando em quatro tratamentos:

- $\quad$ TAA - alto nível de ingestão de energia nas fases de recria e terminação;

- $\quad$ TAM - alto nível de ingestão de energia na fase de recria e médio na fase de terminação;

- TMA - médio nível de ingestão de energia na fase de recria e alto na fase de terminação;

- $\quad$ TMM - médio nível de ingestão de energia nas fases de recria e terminação.

A dieta no confinamento era composta por silagem de sorgo, grão de milho moído, farelo de soja, ureia, monensina sódica, virginiamicina e minerais (Tabela 1). Os ingredientes das dietas foram balanceados (Tabela 2) para atender as exigências 
nutricionais segundo o NRC (1996). A composição da dieta total do tratamento com $50 \%$ de concentrado era de 55,73\% de matéria seca (MS), 16,20\% de proteína bruta (PB), 36,44\% de fibra em detergente neutro (FDN), 22,87\% de fibra em detergente ácido (FDA), 2,27\% de extrato etéreo (EE), 5,63\% de cinzas e $71 \%$ de nutrientes digestíveis totais (NDT) e da dieta com $80 \%$ de concentrado era de $79,75 \%$ de MS, $17,57 \%$ de PB, $17,99 \%$ de FDN, $8,26 \%$ de FDA, $2,22 \%$ de EE, $4,68 \%$ de cinzas, $80 \%$ NDT. Os animais foram alimentados ad libitum, sendo a ração fornecida duas vezes ao dia, às $8 \mathrm{~h}$ e $17 \mathrm{~h}$ de forma a permitir $10 \%$ de sobras. O consumo voluntário foi registrado diariamente, através da pesagem da quantidade de ração oferecida e das sobras do dia anterior, realizada diariamente antes do primeiro arraçoamento da dieta.

Tabela 1. Proporção de ingrediente da ração na base da matéria seca da dieta total dos dois tratamentos da fase de confinamento.

\begin{tabular}{lcc}
\hline \multirow{2}{*}{ Ingredientes (\%) } & \multicolumn{2}{c}{ Proporção de concentrado } \\
Silagem de Sorgo & Médio & Alto \\
\cline { 2 - 3 } Milho triturado & 50,0 & 20,0 \\
Farelo Soja & 29,0 & 66,5 \\
Ureia & 18,5 & 11,5 \\
Mineral* & 0,5 & 0,5 \\
\hline
\end{tabular}

* fosfato bicálcico $13,56 \%$; calcita $50,53 \%$; microminerais $9,86 \%$; cloreto de sódio $24,65 \%$; virginiamicina $0,81 \%$; monensina sódica $0,59 \%$.

Fonte: Elaboração dos autores.

Tabela 2. Composição bromatológica na base da matéria seca do volumoso, concentrado e da dieta total na fase de terminação dos animais em confinamento.

\begin{tabular}{llllll}
\hline & Concentrado & & Silagem & Dieta Total \\
& $50 \%$ & $80 \%$ & Sorgo & $50 \%$ & $80 \%$ \\
\cline { 2 - 6 } MS $^{1}$ & 85,90 & 85,75 & 25,56 & 55,73 & 73,72 \\
PB $^{2}$ & 24,77 & 17,91 & 7,63 & 16,20 & 15,85 \\
FDN $^{3}$ & 13,69 & 13,38 & 59,20 & 36,44 & 22,54 \\
FDA $^{4}$ & 7,14 & 4,60 & 38,60 & 22,87 & 11,40 \\
EE $^{5}$ & 2,63 & 2,21 & 1,90 & 2,27 & 2,15 \\
CINZAS & 7,45 & 4,45 & 3,81 & 5,63 & 4,32 \\
NDT $^{*}$ & - & - & - & 71,00 & 80,00 \\
\hline
\end{tabular}

${ }^{1}$ Matéria seca; ${ }^{2}$ Proteína bruta; ${ }^{3}$ Fibra em detergente neutro; ${ }^{4}$ Fibra em detergente ácido; ${ }^{5}$ Extrato etéreo.

* estimado NDT=40,2625+(0,1969*PB)+(0,4228*ENN)+(1,1903*EE)-(0,1379*FB); Kearl (1982).

Fonte: Elaboração dos autores.

Avaliou-se o consumo de MS e de nutrientes expressos em $\mathrm{kg} / \mathrm{animal} / \mathrm{dia}, \mathrm{kg} / 100 \mathrm{~kg}$ de $\mathrm{PV}$ e por unidade de tamanho metabólico $\left(\mathrm{PV}^{0,75}\right)$. Avaliouse ainda a conversão alimentar (CA) com base no consumo de $\mathrm{kg} / \mathrm{MS} / \mathrm{dia}$ por ganho em peso médio diário.
As pesagens aconteceram no início do confinamento e em intervalos de 21 dias, após jejum de sólidos de 12 horas. Na mesma ocasião foram avaliados os escores corporais em escala de 1 a 5 em que: 1 - estado muito magro e 5 - muito gordo, conforme Restle (1977). 
Os animais foram pesados individualmente sendo o ganho em peso médio diário (GMD) obtido pela diferença entre o peso final e inicial dividido pelo número de dias de alimentação. Na mesma ocasião realizaram-se as mensurações corporais que foram analisadas em função dos parâmetros: inicial, final e de ganho no período total do confinamento. Foram obtidas: altura da cernelha, correspondendo à distância da cernelha até a superfície do solo; altura da garupa, correspondendo à distância do osso sacro até a superfície do solo, sendo estas medidas tomadas com auxílio de bastão graduado e fita métrica; perímetro torácico, que corresponde ao perímetro imediatamente caudal à escápula passando pelo externo e pelos processos espinhais das vértebras torácicas; perímetro de canela, mensurado a partir do diâmetro do osso metacarpiano no membro anterior direito e comprimento, mensurado em linha reta entre a articulação escápulo-umeral e a tuberosidade coxal do ílio tomada lateralmente com auxílio de fita métrica, conforme descrita por Rezende et al. (2011).

$\mathrm{Na}$ análise de variância, para as variáveis estudadas, cada animal constituiu uma unidade experimental. As diferenças entre médias foram detectadas através do teste de Tukey a 5\% de probabilidade. Realizou-se ainda análises de correlação de Pearson.

\section{Resultados e Discussão}

Os animais submetidos ao alto nível de energia da dieta na fase de terminação (TAA e TAM) apresentaram maior $(\mathrm{P}<0,05)$ consumo diário de MS em kg/animal/dia, provavelmente devido ao maior peso dos animais, pois nas demais variáveis expressas em $100 \mathrm{~kg}$ de peso vivo e unidade de tamanho metabólico a diferença entre os tratamentos se mantiveram inalteradas (tabela 3 ).

Não houve diferença $(\mathrm{P}>0,05)$ no consumo de proteína bruta $(\mathrm{CPB})$ e extrato etéreo (CEE) em nenhuma expressão desta variável $(\mathrm{kg} / \mathrm{animal} / \mathrm{dia}$, \%PV e g/PV ${ }^{0,75}$ ) em relação aos tratamentos.

Valores semelhantes foram descritos por Arboitte et al. (2004) estudando a inclusão de diferentes níveis de concentrado em dietas isonitrogenadas obtendo resultados de 1,04; 0,25 e 11,35 para consumo de proteína bruta expressa em $\mathrm{kg} / \mathrm{dia}$ \% $\% \mathrm{PV}$ e por unidade de tamanho metabólico, respectivamente.

Os animais dos tratamentos com 50\% de concentrado na terminação apresentaram maior $(\mathrm{P}<0,05)$ consumo de FDN e FDA expressos em kg /dia e por unidade de tamanho metabólico devido à alta quantidade desses nutrientes na dieta com 50\% de volumoso. Assim como o consumo de NDT, os animais dos tratamentos com $80 \%$ de concentrado apresentaram maior consumo.

Em relação às medidas corporais, não ocorreu diferença $(\mathrm{P}>0,05)$ no comprimento (COMP) inicial entre os animais dos diferentes tratamentos. O comprimento final e no ganho, no entanto, houve interação entre recria e terminação $(\mathrm{P}<0,05$; Tabela 4). Os animais que receberam menor quantidade de energia na recria e terminação apresentaram maior comprimento, provavelmente por permanecerem mais tempo em confinamento. Comparando dois grupos genéticos Nelore e Charolês e seus cruzamentos, Menezes et al. (2008) relataram valores semelhantes aos observados no presente experimento para ganho em comprimento (COMP), sendo 12,67; 13,60 e 8,69 para Charolês, Nelore e mestiços respectivamente. 


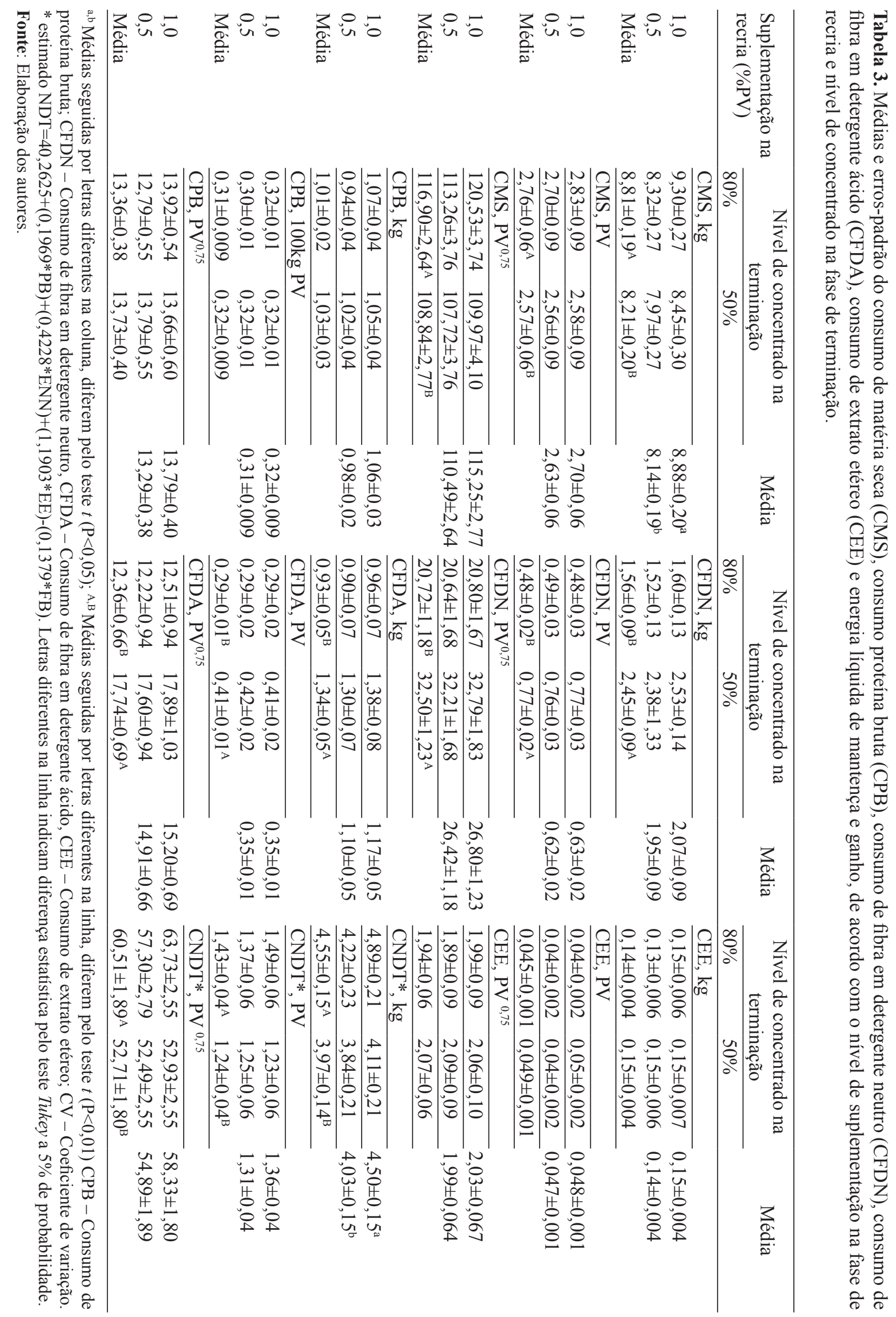


$\mathrm{O}$ perímetro torácico $(\mathrm{PT})$ inicial foi maior $(\mathrm{P}<0,05)$ nos animais suplementados com $1,0 \%$ do peso vivo na fase de recria, entretanto, não ocorreu diferença significativa entre os animais dos tratamentos no PT final. Isso ocorreu devido ao maior $(\mathrm{P}<0,05)$ ganho total desta medida em que antes do confinamento receberam no pasto o nível médio de suplementação. Portanto, a diferença inicial foi compensada no confinamento quando foram terminados com pesos semelhantes. Segundo Rocha et al. (1999), o perímetro torácico (PT) tem sido considerado a medida linear de maior precisão na determinação do crescimento muscular e o comprimento corporal, a de menor precisão, devido a forma de mensurar essas medidas, sendo que o perímetro torácico tem pouca influencia na forma de medir, enquanto que o comprimento pode variar de acordo com a deposição de gordura que afeta os pontos de referência da mensuração. Os mesmos autores observaram que o comprimento e a altura variam em função de crescimento do esqueleto, atingindo um limiar à maturidade, sendo o PT se dá em função do crescimento muscular.

Valores inferiores aos encontrados no presente estudo foram apresentados por Menezes et al. (2008) para ganho em perímetro torácico em animais das raças Charolês, Nelore e mestiços, essa diferença é provavelmente devido a maior densidade energética da dieta no presente estudo.

A altura de cernelha (AC) não diferiu significativamente $(\mathrm{P}>0,05)$ entre tratamentos no início. No final do confinamento os animais que receberam $80 \%$ de concentrado apresentaram maior altura de cernelha. Porém, o ganho total dos animais nos tratamentos médio na recria foram maiores $(\mathrm{P}<0,05)$ que os demais por permanecer mais tempo no confinamento.

Animais que receberam menor nível de suplementação na recria apresentaram menor $(\mathrm{P}<0,05)$ altura de garupa $(\mathrm{AG})$ no início do confinamento, porém, tiveram maior ganho total durante o confinamento pelo mesmo motivo das medidas descritas anteriormente. AG final foi maior $(\mathrm{P}<0,05)$ nos animais dos tratamentos que receberam $50 \%$ de concentrado na terminação.
Northcutt, Wilson e Willham (1992) observaram que mensurações corporais lineares, como altura e comprimento, são mais precisas na determinação do tamanho à maturidade do que o peso. A gordura subcutânea pode sofrer alteração periódica, conforme o estado nutricional dos animais, enquanto as medidas corporais lineares são mais constantes.

Avaliando medidas corporais em cruzamentos entre Nelore e Charolês, Menezes et al. (2008) observaram valores de 6,37; 4,67 e 5,89 cm de ganho em $\mathrm{AC}$ e 7,17; 4,27 e 4,58 cm de ganho em $\mathrm{AG}$, para Charolês, Nelore e mestiços, respectivamente. Houve ganhos inferiores ao presente estudo, provavelmente pelo maior tempo em confinamento, pois os animais que permaneceram menor tempo demonstraram os valores mais próximos.

Animais submetidos ao maior nível de suplementação na recria tiveram maior $(\mathrm{P}<0,05)$ perímetro de canela $(\mathrm{CAN})$ no início da terminação em confinamento. No entanto, não foram observadas diferenças significativas entre os tratamentos para o ganho total e o perímetro de canelafinal, provavelmente em função do crescimento compensatório.

Todas as medidas corporais apresentaram correlação significativa (Tabela 5) com o peso final e não apresentaram para ganho médio diário (GMD). Entre as medidas corporais, o comprimento (COMP) apresentou correlação significativa $(\mathrm{P}<0,01)$ para perímetro torácico $(\mathrm{PT})$, altura de cernelha $(\mathrm{AC})$, altura de garupa (AG) e perímetro de canela (CAN). O PT apresentou correlação significativa e positiva para $\mathrm{AC}$, AG e CAN. AAC apresentou correlação significativa e positiva para AG e CAN. AAG apresentou correlação significativa e positiva para CAN.

Correlações semelhantes foram relatados por Menezes et al. (2008) sendo 0,75; 0,80;0,77; 0,79 para COMP, PT, AC e AG, respectivamente, para o PI, e 0,$80 ; 0,82 ; 0,77 ; 0,73$ de PS para as mesmas características, respectivamente. Houve alta correlação entre as medidas corporais e o peso, principalmente o PT. A utilização na pratica dessas medidas pode ser principalmente para separar lotes na entrada do confinamento e/ou prever dias em confinamentos (REIS et al., 2008). 


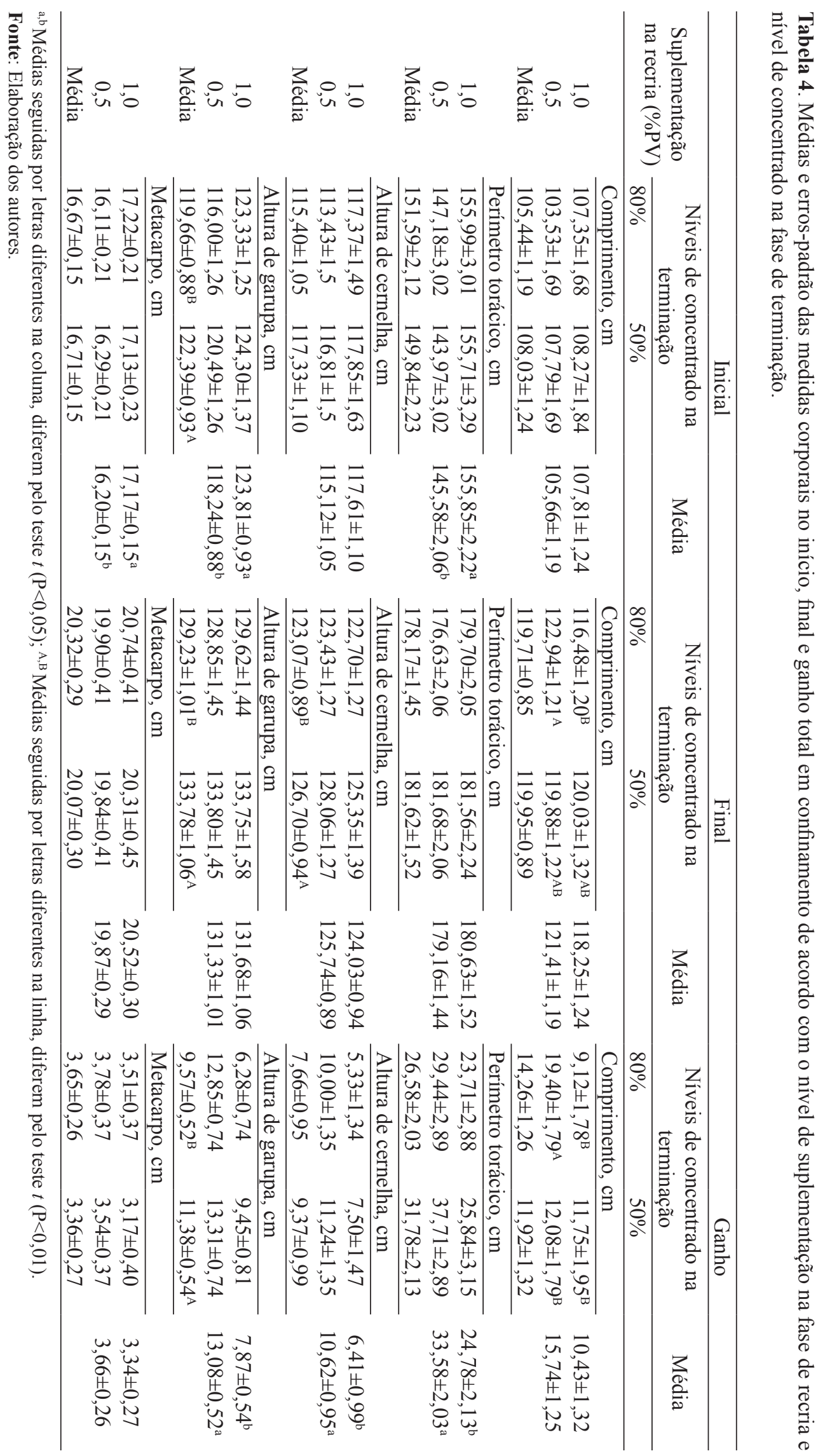


Tabela 5. Coeficiente de correlação entre as variáveis comprimento (COMP), perímetro torácico (PT), altura de cernelha (AC), altura de garupa (AG), perímetro de canela (CAN), ganho médio diário (GMD) e conversão alimentar (CA).

\begin{tabular}{|c|c|c|c|c|c|c|c|}
\hline & COMP & PT & $\mathrm{AC}$ & AG & CAN & GMD & PESO \\
\hline COMP & & $0,64 *$ & $0,70^{*}$ & $0,56^{*}$ & $0,60^{*}$ & 0,15 & $0,72 *$ \\
\hline PT & & & $0,63 *$ & $0,66^{*}$ & $0,73^{*}$ & 0,18 & $0,92 *$ \\
\hline $\mathrm{AC}$ & & & & $0,88^{*}$ & $0,46^{* *}$ & 0,25 & $0,65^{*}$ \\
\hline $\mathrm{AG}$ & & & & & $0,39 * * *$ & 0,25 & 0,71 * \\
\hline CAN & & & & & & 0,13 & 0,81 * \\
\hline GMD & & & & & & & $-0,11$ \\
\hline PESO & & & & & & & \\
\hline
\end{tabular}

$* \mathrm{P}<0,01 ; * * \mathrm{P}<0,05 ; * * * \mathrm{P}<0,1$.

Fonte: Elaboração dos autores.

Avaliando medidas corporais, Cyrillo et al. (2001) observaram correlações genotípicas de 0,$65 ; 0,70$ e 0,48 para altura de garupa, perímetro torácico e comprimento, respectivamente. Valores semelhante foram relatados por Reis et al. (2008) trabalhando com animais do mesmo grupo genético do presente estudo, sendo valores de 0,92; 0,73 e 0,88 para perímetro torácico, comprimento e altura de garupa, respectivamente para correlação com o peso.

O escore corporal (EC) não apresentou diferença ( $P>0,05 ;$ Tabela 6). A concentração energética da dieta tem efeito sobre estado corporal, pois quando atendidas as exigências de manutenção e crescimento, o organismo passa a depositar gordura, portanto, quanto maior a concentração energética da dieta ingerida, maior é a quantidade de energia disponível para deposição de tecido adiposo e mais rápido também será a deposição de gordura subcutânea determinante para conferir o adequado grau de acabamento à carcaça (ARBOITTE et al., 2004).

Comparando diferentes pesos de abate Costa et al. (2002) observaram que o estado corporal e o ganho em estado corporal aumentaram linearmente quando aumentou o peso de abate. Neste estudo os animais abatidos com $340 \mathrm{~kg}$ apresentaram estado corporal final de 3,61, valores superiores ao presente trabalho com o peso de abate superior ao citado pelos autores, sendo essa diferença provavelmente em função do grupo genético mais precoce para deposição de gordura quando comparados a animais mestiços de origem leiteira.

Segundo Menezes e Restle (2005) os animais mestiços Zebuíno x Taurino apresentaram maior escore corporal do que a média dos animais puros, em todas as gerações estudadas. Os autores relataram que a diferença entre o escore corporal dos animais mestiços em relação aos puros reduziu no final do período de confinamento em 40,87\%; 16,99\% e $10,56 \%$ para as gerações 2,3 e 4 , respectivamente.

Os animais dos tratamentos com maiores níveis de energia na recria (TAA e TAM) apresentaram maiores pesos de entrada no confinamento e permaneceram por menos tempo na terminação até atingirem o peso de abate. Estes animais permaneceram 42 e 21 dias a menos no confinamento para TAA e TAM, respectivamente, em relação aos animais dos tratamentos que receberam médio nível de suplementação na recria (TMA e TMM; Tabela 7), evidenciando a importância da suplementação a pasto no peso de entrada no confinamento e conseqüentemente menor período de permanência (PACHECO et al., 2006). 
Tabela 6. Médias e erros-padrão do escore de condição corporal, do peso inicial, ganho em peso total, ganho em peso médio diário e conversão alimentar de acordo com o nível de suplementação na fase de recria e nível de concentrado na fase de terminação.

\begin{tabular}{|c|c|c|c|}
\hline \multirow{2}{*}{$\begin{array}{c}\text { Suplementação na recria } \\
(\% \mathrm{PV})\end{array}$} & \multicolumn{2}{|c|}{ Nível de concentrado na terminação } & \multirow{2}{*}{ Média } \\
\hline & $80 \%$ & $50 \%$ & \\
\hline & \multicolumn{2}{|c|}{ EC, inicial } & \\
\hline 1,0 & $3,23 \pm 0,07$ & $3,15 \pm 0,08$ & $3,19 \pm 0,05$ \\
\hline 0,5 & $3,05 \pm 0,07$ & $3,08 \pm 0,07$ & $3,07 \pm 0,05$ \\
\hline \multirow[t]{2}{*}{ Média } & $3,14 \pm 0,05$ & $3,11 \pm 0,05$ & \\
\hline & \multicolumn{2}{|c|}{ EC, final } & \\
\hline 1,0 & $3,44 \pm 0,04$ & $3,37 \pm 0,05$ & $3,41 \pm 0,03$ \\
\hline 0,5 & $3,39 \pm 0,04$ & $3,38 \pm 0,04$ & $3,39 \pm 0,03$ \\
\hline \multirow[t]{2}{*}{ Média } & $3,42 \pm 0,03$ & $3,38 \pm 0,03$ & \\
\hline & \multicolumn{2}{|c|}{ EC, ganho } & \\
\hline 1,0 & $0,20 \pm 0,05$ & $0,22 \pm 0,05$ & $0,21 \pm 0,039$ \\
\hline 0,5 & $0,33 \pm 0,05$ & $0,30 \pm 0,05$ & $0,32 \pm 0,037$ \\
\hline \multirow{2}{*}{ Média } & $0,27 \pm 0,038$ & $0,26 \pm 0,03$ & \\
\hline & \multicolumn{2}{|c|}{ Peso inicial } & \\
\hline 1,0 & $265,56 \pm 7,33$ & $258,86 \pm 8,03$ & $262,21 \pm 5,43^{\mathrm{a}}$ \\
\hline 0,5 & $224,85 \pm 7,37$ & $228,35 \pm 7,37$ & $226,60 \pm 5,18^{b}$ \\
\hline \multirow[t]{2}{*}{ Média } & $245,21 \pm 5,18$ & $243,60 \pm 5,43$ & \\
\hline & \multicolumn{2}{|c|}{ Ganho em peso total } & \\
\hline 1,0 & $128,73 \pm 7,33$ & $135,44 \pm 8,03$ & $132,08 \pm 5,43^{b}$ \\
\hline 0,5 & $169,44 \pm 7,37$ & $165,94 \pm 7,37$ & $226,60 \pm 5,18^{\mathrm{a}}$ \\
\hline \multirow{2}{*}{ Média } & $149,09 \pm 5,18$ & $150,69 \pm 5,43$ & \\
\hline & \multicolumn{2}{|c|}{ Ganho em peso médio diário } & \\
\hline 1,0 & $1,53 \pm 0,07$ & $1,28 \pm 0,07$ & $1,41 \pm 0,05^{\mathrm{a}}$ \\
\hline 0,5 & $1,38 \pm 0,07$ & $1,32 \pm 0,07$ & $1,35 \pm 0,05^{b}$ \\
\hline \multirow[t]{2}{*}{ Média } & $1,45 \pm 0,05$ & $1,30 \pm 0,05$ & \\
\hline & \multicolumn{2}{|c|}{ Conversão alimentar } & \\
\hline 1,0 & $6,26 \pm 0,43$ & $6,70 \pm 0,47$ & $6,46 \pm 0,31$ \\
\hline 0,5 & $6,11 \pm 0,43$ & $6,04 \pm 0,43$ & $6,08 \pm 0,30$ \\
\hline Média & $6,19 \pm 0,30$ & $6,37 \pm 0,31$ & \\
\hline
\end{tabular}

${ }^{\mathrm{a}, \mathrm{b}}$ Médias seguidas por letras diferentes na coluna, diferem pelo teste $t(\mathrm{P}<0,01)$.

Fonte: Elaboração dos autores.

Tabela 7. Médias dos dias em confinamentos (DC) as médias de peso inicial (PI) e final (PF), ganho em peso total (GPT), ganho médio diário (GMD) e conversão alimentar (CA).

\begin{tabular}{llllll}
\hline & $\mathrm{TAA}^{\mathrm{1}}$ & $\mathrm{TAM}^{2}$ & $\mathrm{TMA}^{3}$ & $\mathrm{TMM}^{4}$ & $\mathrm{CV}^{\mathrm{y}}$ \\
\cline { 2 - 6 } DC & $84,00^{\mathrm{a}}$ & $105,00^{\mathrm{b}}$ & $126,00^{\mathrm{c}}$ & $126,00^{\mathrm{c}}$ & - \\
PI & $263,67^{\mathrm{a}}$ & $259,75^{\mathrm{a}}$ & $224,50^{\mathrm{b}}$ & $224,00^{\mathrm{b}}$ & 10,91 \\
PF & 391,33 & 401,83 & 397,0 & 387,5 & 8,25 \\
GPT & $127,67^{\mathrm{c}}$ & $142,08^{\mathrm{bc}}$ & $172,50^{\mathrm{a}}$ & $163,50^{\mathrm{ab}}$ & 14,67 \\
GMD & 1,5 & 1,4 & 1,4 & 1,3 & 15,47 \\
CA & 6,3 & 6,4 & 6,2 & 6,1 & 17,51 \\
\hline
\end{tabular}

1- Alto nível de concentrado na recria e alto nível de concentrado na terminação; 2-Alto nível de concentrado na recria e médio nível de concentrado na terminação; 3- Médio nível de concentrado na recria e alto nível de concentrado na terminação; 4- Médio nível de concentrado na recria e médio nível de concentrado na terminação.

Fonte: Elaboração dos autores. 
Animais em períodos curtos de confinamento representam menor consumo de energia de mantença. Arboitte et al. (2004) trabalhando com diferentes pesos de abate observaram que o ganho médio diário (GMD) decresceu à medida que o peso de abate aumentou. Observaram também diminuição de $0,450 \mathrm{~kg}$, representando uma redução de $26 \%$ entre os animais com menor peso para os animais com maior peso de abate. Diferença também observada por Costa et al. (2002) de 12,8\% do grupo de $340 \mathrm{~kg}$ de peso para o de $430 \mathrm{~kg}$ peso de abate, explicado pela maior energia gasta para manutenção.

A conversão alimentar (CA) não apresentou diferença significativa $(\mathrm{P}>0,05)$ em relação aos níveis de concentrado nas dietas. Em outro estudo comparando diferentes níveis de concentrado na dieta de animais mestiços, Resende et al. (2001) observaram um comportamento linear decrescente para CA em relação aos níveis de inclusão de 15 , $30,45,60$ e $75 \%$ de concentrado nas dietas. Os autores relataram que a CA melhorou $0,08 \mathrm{~kg} \mathrm{MS} /$ $\mathrm{kg}$ ganho em peso, para cada unidade percentual de aumento do nível de concentrado na dieta. Atribuiuse a este fato, a maior densidade energética da dieta resultante da inclusão crescente de concentrado.

Fernandes et al. (2004) observaram que o desempenho de machos inteiros Nelore $(\mathrm{N}), 1 / 2$ Holandês x Nelore, $1 / 2$ Caracu x Nelore, na fase de recria e terminação, apresentaram similaridade no ganho em peso médio diário e conversão alimentar na fase de terminação com peso de abate fixado em 450 a $480 \mathrm{~kg}$. Outro trabalho com machos de origem leiteira 7/8 e 15/16 Holandês x Zebu, abatidos em três estágios de desenvolvimento, 250, 300 e $350 \mathrm{~kg}$, foi realizado por Rocha et al. (1999), que também observaram ganhos em peso satisfatórios.

O tempo que os animais permanecem no confinamento também pode influenciar a conversão alimentar, como mostraram Arboitte et al. (2004) comparando diferentes pesos de abate. Estes autores observaram que conversão alimentar aumentou linearmente, indicando que a eficiência de transformar alimentos em ganho em peso decresceu. Relataram ainda que os animais abatidos com 425 $\mathrm{kg}$, consumiram 20,4 e 29,6\% menos MS por $\mathrm{kg}$ de ganho de peso do que os abatidos com 467 e 510 $\mathrm{kg}$, respectivamente. Os autores explicaram que a redução na eficiência alimentar foi conseqüência do decréscimo no ganho médio diário provocado pelo aumento do peso de abate de 425 para $510 \mathrm{~kg}$ e que foi causada pelo aumento da exigência da energia liquida de mantença (Elm) e pela composição de ganho em peso, que passou a ser mais energética à medida que o peso de abate aumentou.

\section{Conclusão}

O maior peso de entrada dos animais previamente suplementados com alto nível de energia resulta em menor tempo de permanência no confinamento permitindo concluir que esta estratégia alimentar é tecnicamente mais atrativa. Mais estudos devem ser realizados abordando a eficiência econômica de diferentes sistemas de alimentação de machos mestiços de origem leiteira, nas diferentes fases, com a finalidade de produção de carne de qualidade de maneira eficiente.

\section{Referências}

ARBOITTE, M. Z.; RESTLE, J.; ALVES FILHO, D. C. A.; BRONDANI, I. L.; SILVA, J. H. S.; NÖRNBERG, J. L.; KUSS, F. Desempenho em confinamento de novilhos $5 / 8$ Nelore $-3 / 8$ Charolês abatidos em diferentes estádios de desenvolvimento. Revista Brasileira de Zootecnia, Viçosa, v. 33, n. 4, p. 947-958, 2004.

COSTA, E. C.; RESTLE, J.; PASCOAL, L. L.; VAZ, F. N.; ALVES FILHO, D. C.; ARBOIITTE, M. Z. Desempenho de novilhos Red Angus superprecoçes, confinados e abatidos com diferentes pesos. Revista Brasileira de Zootecnia, Viçosa, v. 31, n. 1, p. 129-138, 2002.

CYRILLO, J. N. S. G.; RAZOOK, A. G.; FIGUIREDO, L. A.; BONILHA NETO, L. M.; MERCADANTE, M. E. Z.; TONHATI, H. Estimativas de tendências e parâmetros genéticos do peso padronizado aos 378 dias de idade, medidas e perímetro escrotal de machos Nelore 
de Sertãozinho, SP. Revista Brasileira de Zootecnia, Viçosa, v. 30, n. 1, p. 56-65, 2001.

ESCOLA DE AGRONOMIA DA UNIVERSIDADE FEDERAL DE GOIÁS - EA/UFG. Estação meteorológica. Goiânia, 2009. Disponível em: $<$ http:// agro.ufg.br/pages/63545-estacao-evaporimetrica> . Acesso em: 02 jun. 2009.

FERNANDES, J. F.; PAULINO, M. F.; MARTINS, R. G. R.; VALADARES FILHO, S. C.; TORRES, R. A.; PAIVA, L. M.; MORAES, G. F. B. K. Ganho de peso, conversão alimentar, ingestão diária de nutrientes e digestibilidade de garrotes não castrados de três grupos genéticos em recria e terminação. Revista Brasileira de Zootecnia, Viçosa, v. 33, n. 6, p. 2403-2411, 2004. Suplemento 3.

MENEZES, L. F. G.; RESTLE, J. Desempenho de novilhos de gerações avançadas do cruzamento alternado entre as raças Charolês e Nelore, terminados em confinamentos. Revista Brasileira de Zootecnia, Viçosa, v. 34, n. 6, p. 1927-1937, 2005.

MENEZES, L. F. G.; RESTLE, J.; KUSS, F.; BRONDANI, I. L.; ALVES FILHO, D. C.; CATELLAM, J.; OSMARI, M. P. Medidas corporais de novilhos das gerações avançadas do cruzamento rotativo entre as raças Charolês e Nelore, terminados em confinamento. Ciência Rural, Santa Maria, v. 38, n. 3, p. 771-777, 2008.

NATIONAL RESEARCH COUNCIL - NRC. Nutrient requirements of domestic animals. $7^{\text {td }}$ ed. Washington: National Academy Press, 1996. 242 p.

NORTHCUTT, S. L.; WILSON, D. E.; WILLHAM, R. L. Adjusting weight for boby condition score in Angus cows. Journal of Animal Science, Champaign, v. 70, n. 5, p. 1342-1345, 1992.

PACHECO, P. S.; RESTLE, J.; VAZ, F. N.; FREITAS, A. K.; PADUA, J. T.; NEUMANN, M.; ARBOITTE, M. Z. Apreciação econômica da terminação em confinamento de novilhos jovens e superjovens de diferentes grupos genéticos. Revista Brasileira de Zootecnia, Viçosa, v. 35, n. 1, p. 309-320, 2006.
PORTO, M. O.; PAULINO, M. F.; VALADARES FILHO, S. C.; SALES, M. F. L.; DETMANN, E.; CAVALI, J. Formas de utilização do milho em suplemento para novilhos na fase de terminação em pastagem no período das águas: desempenho e parâmetros nutricionais. Revista Brasileira de Zootecnia, Viçosa, v. 37, n. 12, p. 2251-2260, 2008.

REIS, G. L.; ALBUQUERQUE, F. H. M. A. R.; VALENTE, B. D.; MARTINS, G. A.; TEODORO, R. L.; FERREIRA, M. B. D.; MONTEIRO, J. B. N.; SILVA, M. A.; MADALENA, F. E. Predição do peso vivo a partir de medidas corporais em animais mestiços Holandês x Gir. Ciência Rural, Santa Maria, v. 38, n. 3, p. 778-783, 2008.

RESENDE, F. D.; QUEIROZ, A. C.; OLIVEIRA, J. V.; PEREIRA, J. C.; MÂNCIO, A. B. Bovinos mestiços alimentados com diferentes proporções de volumoso: concentrado. 1. digestibilidade aparente dos nutrientes, ganho de peso e conversação alimentar. Revista Brasileira de Zootecnia, Viçosa, v. 30, n. 1, p. 261-269, 2001.

RESTLE, J. Efeito da suplementação com energia e energia-proteína no ganho de peso e no comportamento reprodutivo de vacas. Revista da Faculdade de agronomia da UFRGS, Porto Alegre, v. 1, n. 2, p. 203-204, 1977.

REZENDE, P. L. P.; RESTLE, J.; RESENDE, J. J. R.; PÁDUA, J. T.; FREITAS NETO, M. D. F.; ROCHA, F. M. Desempenho e desenvolvimento corporal de bovinos leiteiros mestiços submetidos a níveis de suplementação em pastagem de Brachiaria Brizantha. Ciência Rural, Santa Maria, v. 41, n. 8, p. 1453-1458, 2011.

ROCHA, E. O.; FONTES, C. A. A.; PAULINO, M. F.; LADEIRA, M. M. Ganho de peso, eficiência alimentar e características de carcaça de novilhos de origem leiteira. Revista Brasileira de Zootecnia, Viçosa, v. 28, n. 1, p. 148-159, 1999. 